PICTORIAL ESSAY

\title{
Role of FDG PET/CT in imaging of renal lesions
}

\author{
R Kochhar, ${ }^{1}$ RK Brown, ${ }^{2}$ CO Wong, ${ }^{3}$ NR Dunnick, ${ }^{2}$ KA Frey ${ }^{2}$ and P Manoharan ${ }^{1}$ \\ ${ }^{1}$ Department of Radiology, The Christie, NHS Foundation Trust, Manchester, UK; and 2Diagnostic Radiology, Division of Nuclear Medicine, University of \\ Michigan, Ann Arbor, and ${ }^{3}$ Department of Nuclear Medicine, William Beaumont Hospitals, Royal Oak, Michigan, USA
}

\begin{abstract}
R Kochhar MBBS, MD, FRCR; RK Brown MD; CO Wong MBBS, PhD, MD; NR Dunnick MD; KA Frey MD, PhD; P Manoharan MBChB, MRCP, FRCR.
\end{abstract}

\section{Correspondence \\ Dr Prakash Manoharan, Consultant Radiologist, Department of Radiology, The Christie NHS Foundation Trust, Wilmslow Road, Manchester M20 4BX, UK. \\ Email: prakash.manoharan@christie.nhs.uk}

Conflict of interest: None.

Submitted 1 June 2009; accepted 18 May 2010.

doi:10.1111/j.1754-9485.2010.02181.x

\begin{abstract}
Summary
Focal incidental renal lesions are commonly encountered on positron emission tomography (PET)/computed tomography (CT) imaging. The vast majority of these lesions are benign. However, the interpretation of renal lesions can be problematic if the imaging criteria of simple cysts are not met. Limited literature exists on the characterisation of renal masses with metabolic imaging. The purpose of this article is to focus on the imaging features of benign and malignant renal masses with PET/CT. The lesions discussed include renal cyst, angiomyolipoma, oncocytoma, renal cell carcinoma, renal metastases and other infiltrating neoplastic processes affecting the kidney. Both the anatomical and metabolic features which characterise these benign and malignant entities are described. We emphasise the importance of viewing the $\mathrm{CT}$ component to identify the typical morphological features and discuss how to best use hybrid imaging for management of renal lesions. Metabolic imaging has a promising role in the imaging of renal lesions and can help prevent unnecessary biopsies and ensure optimal management of suspicious lesions.
\end{abstract}

Key words: computed tomography; diagnostic imaging; positron emission tomography; renal neoplasm; uroradiology.

\section{Introduction}

Hybrid imaging with positron emission tomography (PET)/ computed tomography (CT) is routinely used in the assessment of malignant disease. The vast majority of PET/CT scans are performed with fluorine-18 fluoro2-deoxy-D-glucose (FDG), an analogue of glucose. There is significant uptake and excretion of FDG through the kidneys which results in intense activity in the renal collecting system and the bladder (Fig. 1). The use of FDG PET/CT is thus limited in the evaluation of genitourinary lesions. However, the renal parenchyma is a common site of incidental findings on hybrid imaging. Limited literature exists on the imaging appearances and characterisation of renal masses with metabolic imaging. This pictorial review illustrates both the anatomical and metabolic features of a wide spectrum of renal masses on PET/CT and discusses the current role of hybrid $\mathrm{PET} / \mathrm{CT}$ in the imaging of renal lesions. All illustrated solid lesions were histologically confirmed except for a suspected renal metastasis in the native kidney postrenal transplant.

\section{Renal lesions on FDG PET/CT}

\section{Renal cyst}

Renal cysts are the most commonly encountered renal lesions, and their prevalence increases with increasing age, male gender, renal dysfunction and hypertension. ${ }^{1}$ Renal cystic lesions are usually classified using the Bosniak criteria which is an internationally recognised classification (Table 1 ). The typical appearance of a simple cyst on CT is of a well-defined, thin walled, low attenuation lesion (0-20 HU). On hybrid imaging with FDG PET/CT, simple renal cysts are photopenic (Fig. 2). Simple renal cysts do not need PET/CT for characterisation and do not require follow up. The challenge is with complex cysts and FDG PET/CT has a role for the noninvasive characterisation of indeterminate cysts (Bosniak 


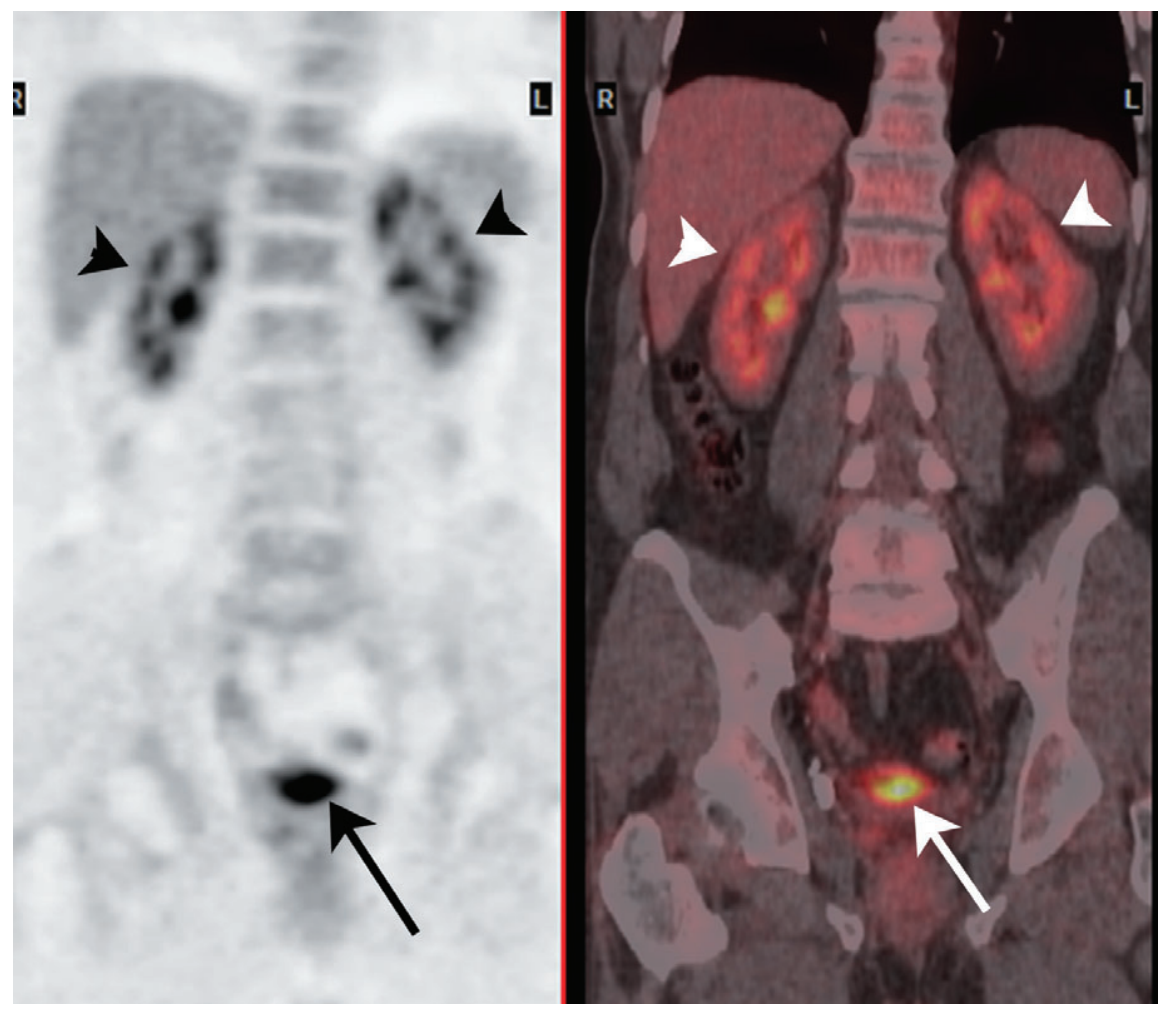

Fig. 1. Normal fluorine-18 fluoro-2-deoxy-Dglucose (FDG) excretion. Coronal attenuated corrected positron emission tomography (PET) image (left) and fused PET/CT image (right) demonstrate normal FDG excretion in bilateral renal collecting systems (arrow heads) and bladder (arrows).

Types IIF and III) (Fig. 3). In a study by Goldberg et al., PET correctly classified indeterminate cysts as benign in 7 of the 8 patients confirmed by surgery or needle aspiration, and a negative PET scan in conjunction with a negative cyst aspiration offers confirmatory evidence of benignity. ${ }^{2}$ PET/CT can also help in precise anatomic localisation and percutaneous drainage of an infected cyst in autosomal dominant polycystic kidney disease because these are difficult to localise with conventional imaging. ${ }^{3}$

Table 1. Bosniak classification of renal cystic disease

\begin{tabular}{|c|c|c|}
\hline Bosniak category & Imaging features & Role of PET/CT \\
\hline Category I & $\begin{array}{l}\text { Simple benign cysts showing homogeneity, water content and a sharp interface with } \\
\text { adjacent renal parenchyma, with no wall thickening, calcification or enhancement }\end{array}$ & $\begin{array}{l}\text { No role in characterisation } \\
\text { Photopenic lesions }\end{array}$ \\
\hline Category II & $\begin{array}{l}\text { Cystic lesions with one or two thin ( } \leq 1 \mathrm{~mm} \text { thick) septations or thin, fine calcification in } \\
\text { their walls or septa (wall thickening }>1 \mathrm{~mm} \text { advances the lesion into surgical category } \\
\text { III) and hyperdense benign cysts with all the features of category I cysts except for } \\
\text { homogeneously high attenuation }\end{array}$ & $\begin{array}{l}\text { No role in characterisation } \\
\text { Potential role in precise localisation and } \\
\text { drainage of infected cysts }\end{array}$ \\
\hline Category II F & $\begin{array}{l}\text { Minimally complicated cysts that need follow-up. This is a group not well defined by } \\
\text { Bosniak but consists of lesions that do not neatly fall into category II. These lesions } \\
\text { have some suspicious features that deserve follow-up up to detect any change in } \\
\text { character. }\end{array}$ & $\begin{array}{l}\text { Potential role in non-invasive characterisation } \\
\text { Potential role in precise localisation and } \\
\text { drainage of infected cysts }\end{array}$ \\
\hline Category III & $\begin{array}{l}\text { True indeterminate cystic masses that need surgical evaluation, although many prove to } \\
\text { be benign. They may show uniform wall thickening, nodularity, thick or irregular } \\
\text { peripheral calcification, or a multilocular nature with multiple enhancing septa. } \\
\text { Hyperdense lesions that do not fulfil category II criteria are included in this group. }\end{array}$ & $\begin{array}{l}\text { Useful for non-invasive characterisation } \\
\text { Negative PET in conjunction with negative cyst } \\
\text { aspiration offers confirmatory evidence of } \\
\text { benignity }\end{array}$ \\
\hline Category IV & $\begin{array}{l}\text { Lesions with a non-uniform or enhancing thick wall, enhancing or large nodules in the } \\
\text { wall, or clearly solid components in the cystic lesion. } \\
\text { Enhancement was considered present when lesion components increased by at least } \\
10 \mathrm{HU} \text {. }\end{array}$ & $\begin{array}{l}\text { Lesion would be best characterised with } \\
\text { contrast enhanced CT/MR and biopsy } \\
\text { confirmation. } \\
\text { Role in restaging and metastatic disease }\end{array}$ \\
\hline
\end{tabular}

PET, positron emission tomography. 

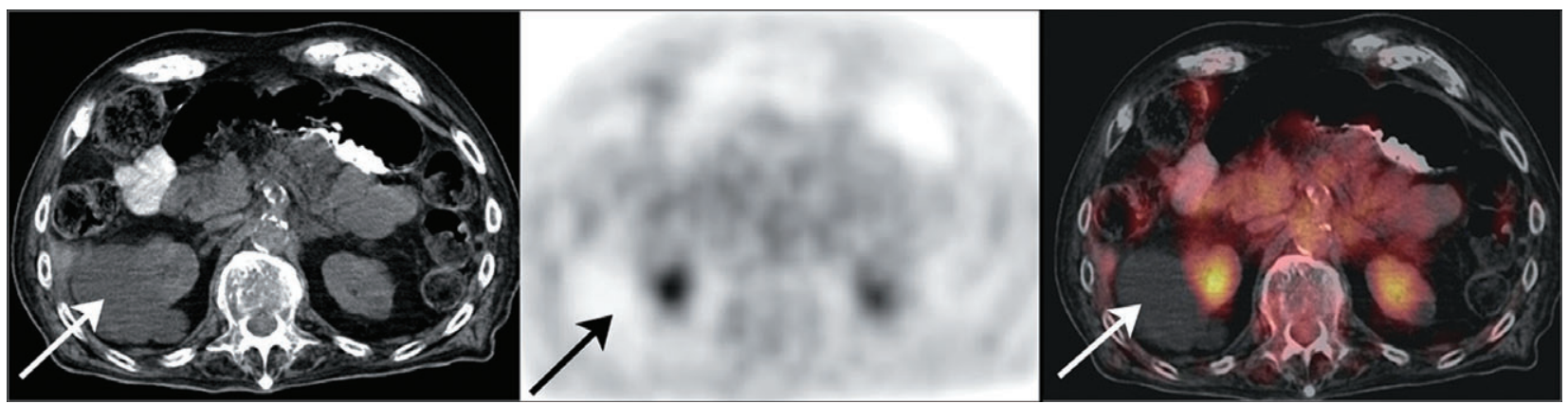

Fig. 2. Fluorine-18 fluoro-2-deoxy-D-glucose (FDG) positron emission tomography (PET)/CT appearances of simple renal cyst. Unenhanced CT component (left) demonstrates well-defined right renal cysts with fluid attenuation (arrow). FDG PET image (centre) and fused PET/CT image (right) demonstrate corresponding central area of photopenia (arrows), consistent with simple renal cysts. Note also a small simple cyst in the left kidney.

\section{Renal angiomyolipoma}

Angiomyolipoma ( $\mathrm{AML}$ ) is the most common benign tumour of the kidney. It is usually asymptomatic, although life-threatening haemorrhage can occur. Most lesions are identified as an incidental finding or following imaging studies undertaken to investigate haematuria. In approximately $10 \%$ of patients, AML develops as part of the tuberous sclerosis complex; there is also an association with sporadic lymphangioleiomyomatosis. ${ }^{4}$ These lesions characteristically contain variable amounts of abnormal blood vessels, adipose tissue and smooth muscle elements. In about $5 \%$ of the lesions, a predominantly solid neoplasm composed of smooth muscle is seen. The vast majority of AML can be accurately diagnosed on unenhanced CT as the lesions contain macroscopic fat. Infrequently, a large renal cell carcinoma (RCC) may contain a small amount of adipose tissue, making it difficult to differentiate from an AML. AML rarely contains calcification; therefore, if a lesion has calcium and fat, RCC must be considered as the likely diagnosis. $^{5}$

There is limited literature on the role of FDG PET/CT in the diagnosis of AML. Of the published data, description has been variable with one author documenting AML as metabolically active on FDG PET and falsely positive for
RCC. ${ }^{6}$ Another group assessing a focal liver mass found that AML did not have increased FDG uptake but had increased ${ }^{11} \mathrm{C}$ acetate uptake, which they attributed to the smooth muscle uptake in that lesion. ${ }^{7}$ The AML lesion we encountered did not demonstrate an increased FDG uptake (Fig. 4) and as such, PET/CT has limited role characterisation because of the variable uptake. In the majority of patients, the CT appearances would be the major criteria in assessing renal AML. However, in patients where AML develops as a part of the tuberous sclerosis complex, FDG PET is helpful and can clearly demonstrate and localise tubers in the brain as hypometabolic regions compared with the FDG uptake in the normal brain. ${ }^{8}$

\section{Renal oncocytoma}

Renal oncocytoma are predominantly solid renal tumours, thought to be benign. They account for 2-3\% of renal tumours, are usually asymptomatic and can be an incidental finding. ${ }^{9}$ On unenhanced $\mathrm{CT}$, they usually appear isodense or hypodense to renal parenchyma. The mass shows homogenous enhancement which is generally lower than the surrounding renal parenchyma. If a central stellate scar is present, it is hypodense on both pre- and post-contrast images.
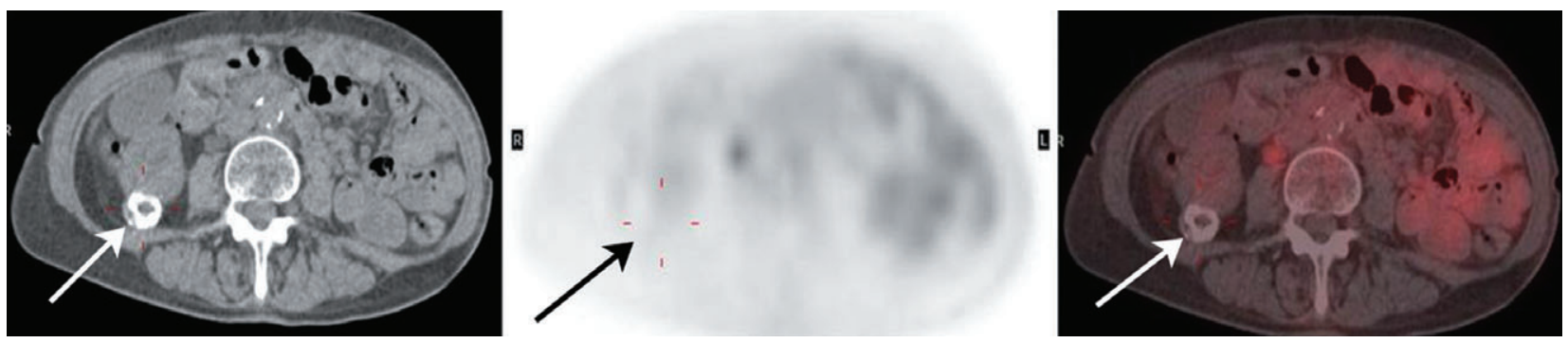

Fig. 3. Fluorine-18 fluoro-2-deoxy-D-glucose (FDG) positron emission tomography (PET)/CT of a complex renal cyst. Unenhanced CT component (left) demonstrates a complex Bosniak category II/III cyst in the right kidney with a thick calcific wall (arrow). FDG PET image (centre) and fused PET/CT image (right) demonstrate no FDG uptake in the lesion (arrows). Follow-up was recommended but biopsy/aspiration was not performed. 


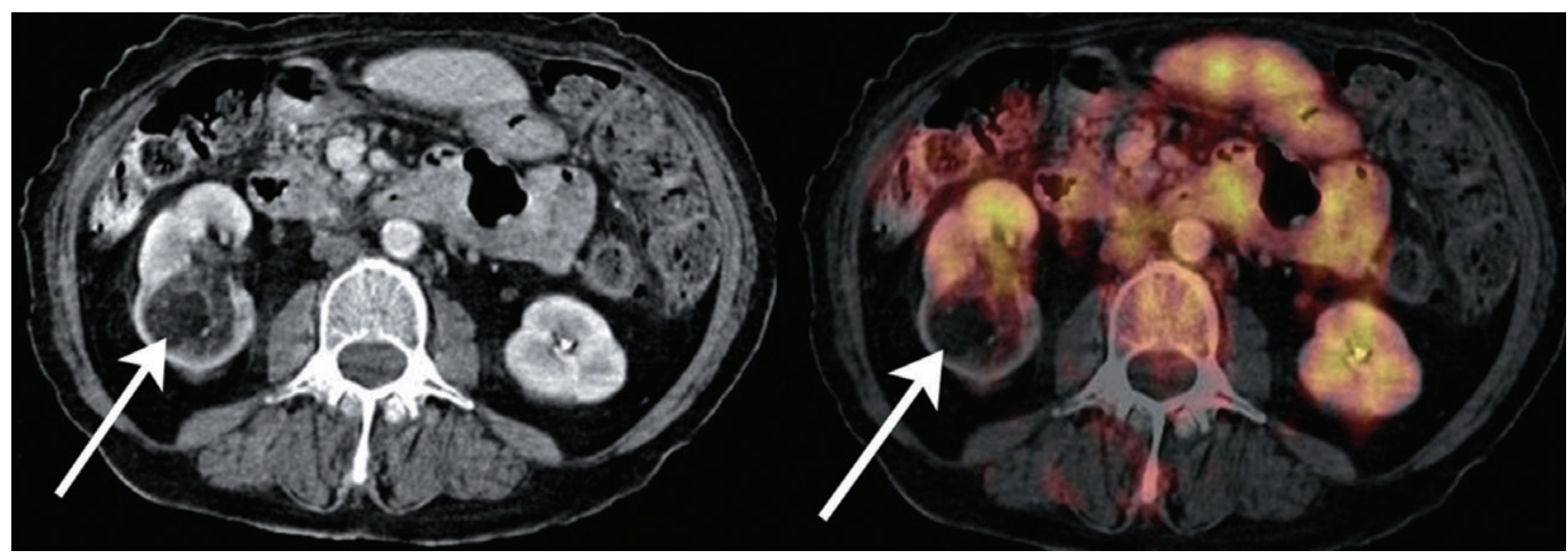

Fig. 4. Fluorine-18 fluoro-2-deoxy-D-glucose (FDG) positron emission tomography (PET)/CT appearances of renal angiomyolipoma (AML). Contrast-enhanced CT image (left) and fused PET/CT image (right) demonstrate a heterogeneous attenuation lesion with fat attenuation in the lower pole of the right kidney on CT (arrow) with minimal uptake of FDG on fused PET/CT image (arrow). The lesion has typical CT characteristics of renal AML which are known to have a variable FDG uptake, confirmed on histopathology.

Both oncocytoma and RCC are treated surgically. In fact, recent articles have reported cases of local recurrence and even metastases following resection of oncocytoma. ${ }^{10}$ These observations suggest that oncocytoma could represent the benign form of malignant chromophobe cell tumours such as RCC. ${ }^{9}$

As with $A M L$, the published reports on the FDG appearances of oncocytoma are also limited. A recent case report described an oncocytoma that had significant uptake of FDG; ${ }^{11}$ however, oncocytoma may not have any appreciable uptake of FDG (Fig. 5), limiting the usefulness of FDG PET/CT in characterisation. The diagnostic conundrum that usually arises is the differentiation between oncocytoma and RCC, and metabolic imaging may not be helpful in differentiating these lesions. ${ }^{12}$

\section{Renal cell carcinoma}

Worldwide renal cancer accounts for nearly $2 \%$ of all cancers and increases in kidney cancer incidence have been reported in many different countries around the world. ${ }^{13}$ The current classification of RCC describes four major types, namely: clear cell, chromophil (papillary), chromophobe and collecting duct type, with the clear cell RCC being by far the most common type. ${ }^{14}$ Pre-operative staging is accurately performed with contrast enhanced CT. RCC can appear hypodense, isodense or hyperdense on pre-contrast studies. It usually enhances significantly post-contrast and can be heterogeneous because of tumour necrosis.

Only a limited number of studies have investigated the utility of PET in the assessment of renal masses and
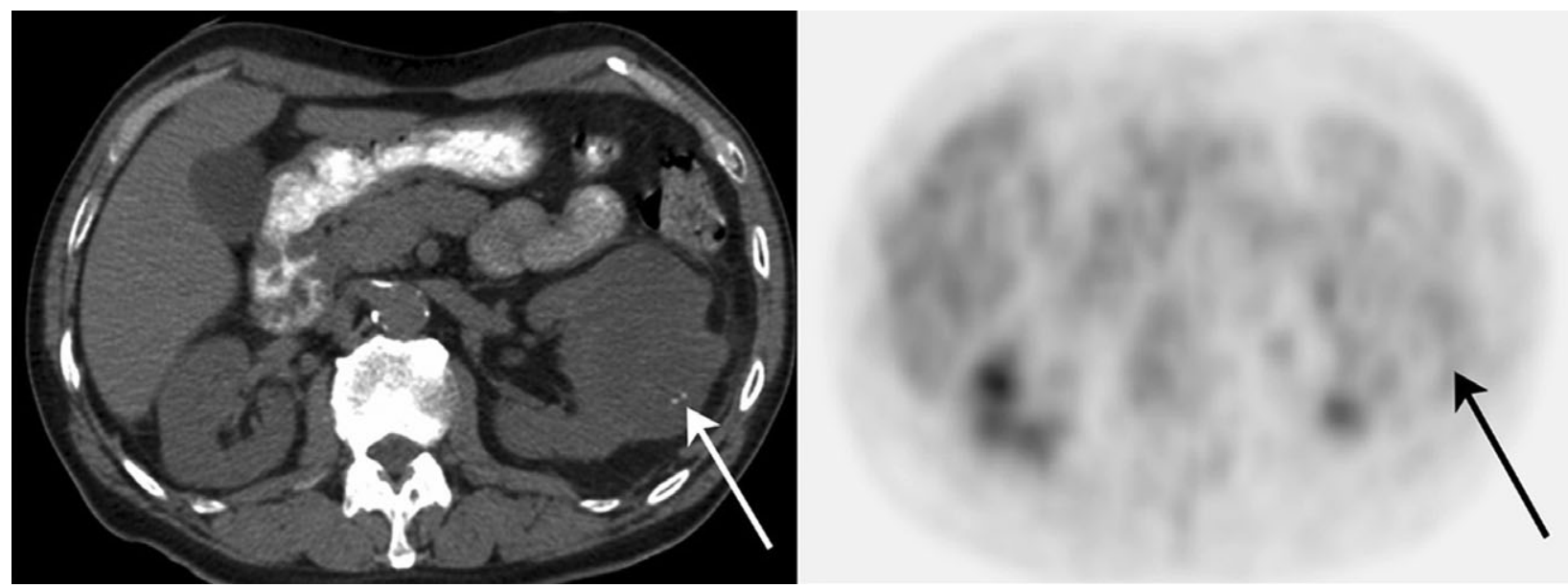

Fig. 5. Fluorine-18 fluoro-2-deoxy-D-glucose (FDG) positron emission tomography (PET)/CT appearances of a renal oncocytoma. Unenhanced CT image (left) demonstrates a hypodense soft tissue lesion with tiny specks of calcification (arrow) in the mid pole of the left kidney. Corresponding PET image (right) demonstrates only minimal heterogeneous uptake of FDG in the lesion. The lesion was a histologically confirmed renal oncocytoma. 

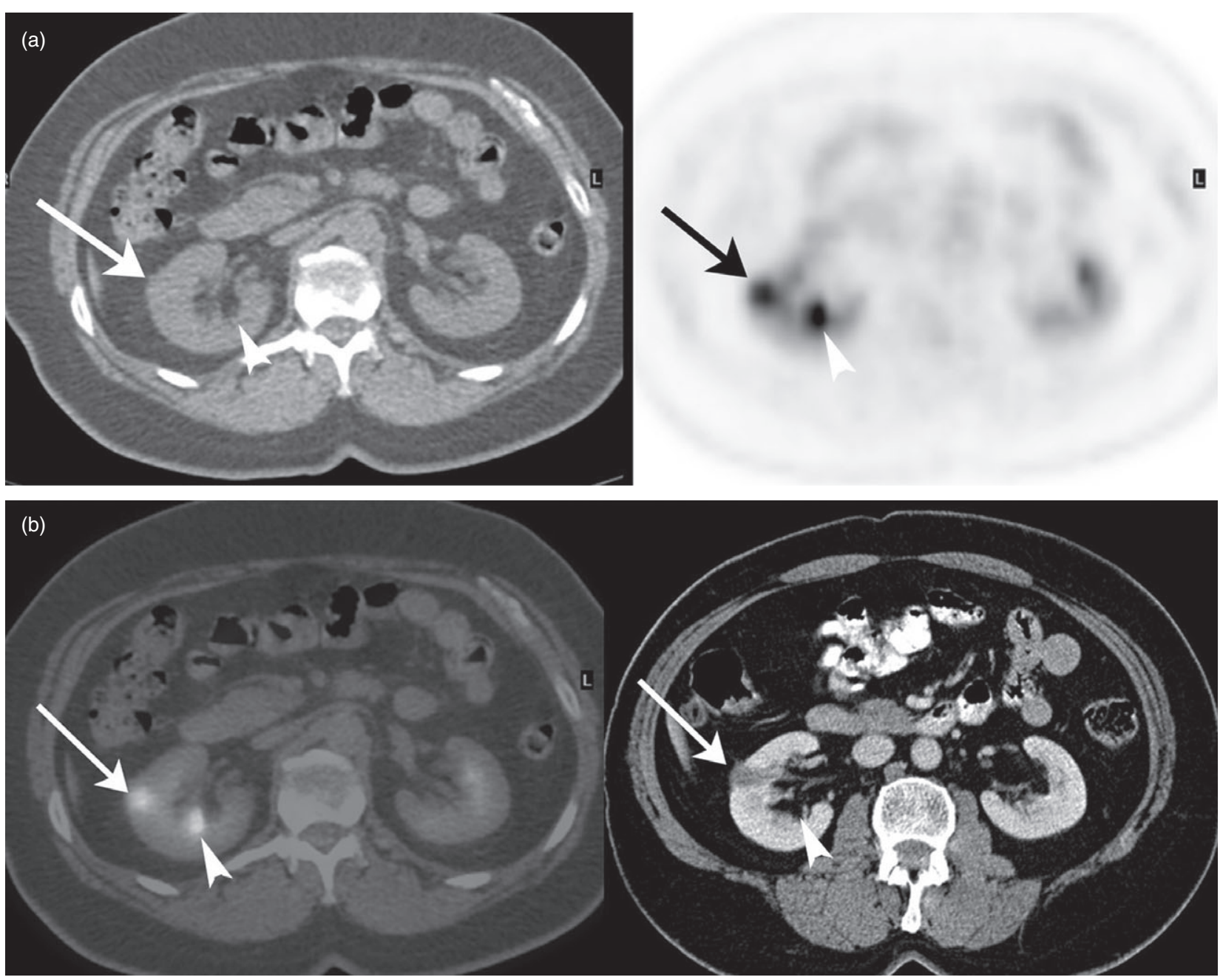

Fig. 6. Fluorine-18 fluoro-2-deoxy-D-glucose (FDG) positron emission tomography (PET)/CT appearances of a renal cell (RCC) carcinoma with high-grade uptake. (a) Unenhanced CT component (left) demonstrates a barely perceivable isodense right renal lesion (arrow). The PET image (right) demonstrates focal increased FDG uptake (arrow) which can be easily mistaken to represent normal uptake in the collecting system (arrow head). (b) Fused PET/CT image (left) clearly localises the uptake to the renal cortex (arrow) and the adjacent focus of uptake lies within the collecting system (arrow head). Contrast-enhanced CT image (right) confirms the presence of a small renal tumour in the right kidney (arrow) which showed high-grade FDG uptake and normal appearances for the collecting system (arrow head). RCC was histopatholically confirmed post-partial right nephrectomy.

primary staging of RCC. Bachor et al. studied 29 patients with solid renal masses and found that PET was true positive in 20 of 26 patients (77\%) with confirmed RCC. ${ }^{6}$ In one of the larger series on clinical use of PET in detection of RCC, FDG PET exhibited a sensitivity of $60 \%$ and specificity of $100 \%$ for primary RCC, which was unfavourable to abdominal CT ( $92 \%$ sensitivity and $100 \%$ specificity)..$^{15}$ The authors concluded that the role of FDG PET in the detection of RCC is limited by low sensitivity. This has been supported by another study where FDG PET performed for both characterisation and staging of suspicious renal masses, in a subgroup of 35 patients, showed high false negative rates, leading to a sensitivity, specificity and accuracy of 47,80 and $51 \%$, respectively ${ }^{16}$.
This is partly because of the excretion of FDG via the urinary tract as well as the significant variability in the uptake of FDG by RCC. $6,17,18$ Various authors have postulated causes for this inconsistency, including the variable expression of GLUT- 1 and the lack of availability of FDG in large tumours as a result of central necrosis. However, PET-positive tumours have higher tumour grade and increased GLUT-1. ${ }^{17}$ In our experience, FDG uptake in RCC can range from uptake similar to the background activity to intensely FDG avid lesions (Figs 6-8). Overall, FDG PET has not greatly improved the detection and management of primary renal cancer because the sensitivity and specificity of this technique seem to be less effective than CT. ${ }^{19}$ 

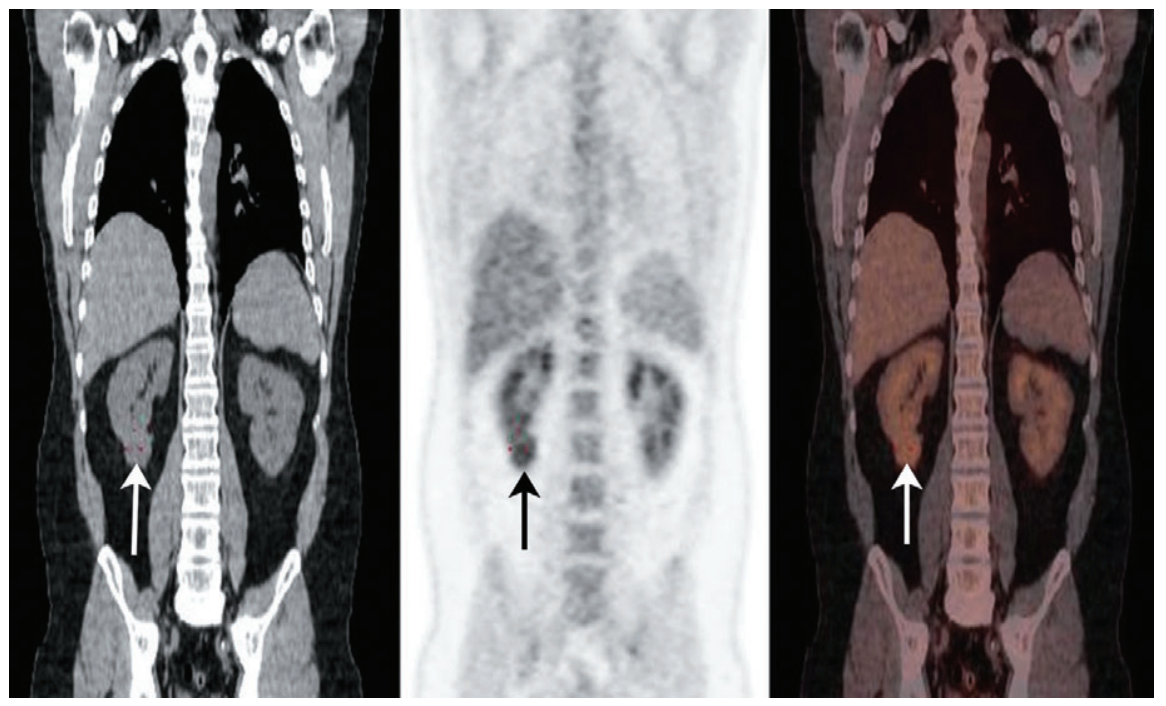

Fig. 7. Fluorine-18 fluoro-2-deoxy-D-glucose (FDG) positron emission tomography (PET)/CT appearances of a renal cell carcinoma (RCC) with intermediate grade uptake. Unenhanced CT image (left) demonstrates an exophytic lesion seen in the lower pole of the right kidney (arrow). PET image (centre) and fused PET/CT image (right) demonstrate low grade uptake in the lesion (arrows) similar to background renal uptake. This was a histologically confirmed RCC showing only low to intermediate grade FDG uptake.

A meta-analysis of performance of FDG PET in RCC concluded that PET has a limited role in detection of the primary disease; however, it can be useful in restaging and detection of metastatic disease. ${ }^{20}$ In particular, $\mathrm{PET} / \mathrm{CT}$ is useful for lymph node metastases which can often be falsely negative using the CT size criteria. ${ }^{16,18}$ FDG PET/CT has been shown to be as good as conventional methods, with $85.7 \%$ accuracy in detecting recurrence or metastases. ${ }^{21}$ In addition, it is possible to examine all organ systems in one procedure with no need for contrast agents, which can be detrimental to renal function.

There has been a recent impetus to ascertain tumour histology before definitive surgery in primary RCC. FDG PET cannot differentiate between clear cell and papillary renal cancers. However, Divgi and colleagues have shown that PET imaging of renal masses, using the 124I-labelled chimeric antibody G250 (124I-G250), is able to identify correctly clear-cell tumours of the kidney in most of the patients studied (sensitivity 94\%, specificity $100 \%) .{ }^{22}$ This study shows for the first time that molecular imaging with PET has a role in renal cancer. ${ }^{19}$

\section{Renal lymphoma and leukaemia}

Because the kidneys do not contain lymphoid tissue, primary renal lymphoma is a rare disease, representing only $0.1-0.7 \%$ of extranodal lymphoma. ${ }^{23}$ Renal involvement is, however, commonly encountered in patients with disseminated non-Hodgkin's lymphoma and is detected in $3-8 \%$ of all patients undergoing routine CT imaging. ${ }^{24}$ There are a number of typical radiological patterns of renal lymphoma, namely: multiple renal masses, solitary masses, renal invasion from contiguous retroperitoneal disease, perirenal disease and diffuse renal infiltration. The commonest appearance on
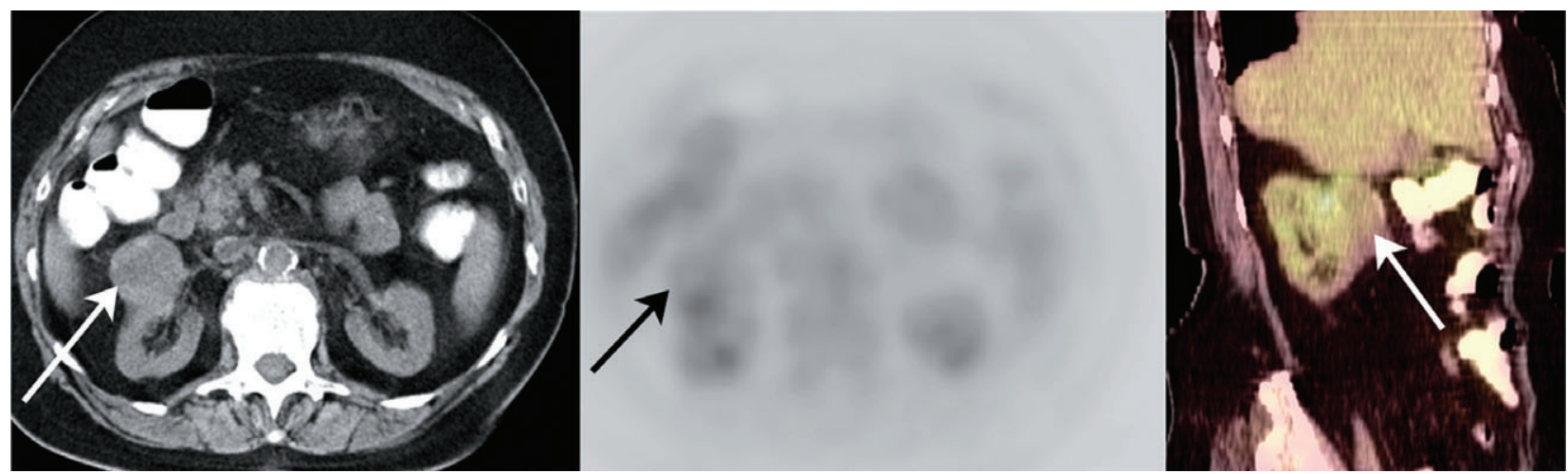

Fig. 8. Fluorine-18 fluoro-2-deoxy-D-glucose (FDG) positron emission tomography (PET)/CT appearances of a renal cell carcinoma (RCC) with very low-grade uptake. Unenhanced CT (left) shows a solid exophytic mass in the mid pole of the right kidney. FDG PET image (centre) and fused PET/CT image (left) demonstrate that the mass shows minimal FDG uptake, less than the background renal uptake. The lesion was a histologically confirmed RCC with very low grade uptake. 

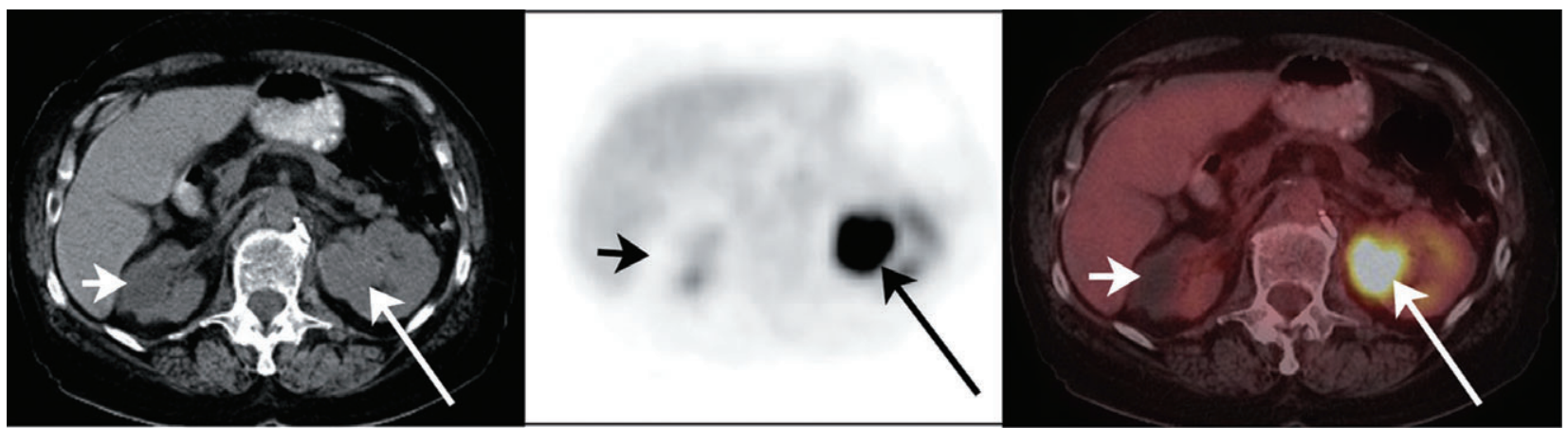

Fig. 9. Fluorine-18 fluoro-2-deoxy-D-glucose (FDG) positron emission tomography (PET)/CT appearances of renal lymphoma. Unenhanced CT image (left) demonstrates an isodense lesion in the left kidney (arrow) and fluid attenuation cystic lesion in the right kidney (short arrow). PET image (centre) and fused PET/CT image (right) demonstrate markedly increased uptake of FDG in the lesion in the left kidney, in keeping with a metabolically active histologically confirmed lymphomatous lesion (arrows). The cystic lesion in the right kidney is photopenic (short arrow), typical for a simple cyst.

contrast-enhanced CT is of multiple bilateral low attenuation renal masses, typically $1-3 \mathrm{~cm}$ in size.

There is a paucity of literature describing the appearances of renal involvement in lymphoma on FDG PET/CT. The discrepancy between the appearances of a distorted renal parenchymal outline with foci of increased uptake as opposed to the normal collecting system uptake of FDG PET should alert the reporting physician. A careful review of the CT and fused images helps to accurately locate the site of activity within the renal parenchyma (Fig. 9).

Contiguous retroperitoneal and perirenal pathology are also seen. Large retroperitoneal masses can invade and displace the renal hilar structures. These masses do not typically resolve following therapy; hence, the role of metabolic imaging in detecting disease recurrence is of paramount importance. The perirenal pattern could either be because of disease arising in perinephric lymph nodes, direct retroperitoneal extension or extension from the renal parenchyma. These lesions appear as low attenuation areas on contrast $\mathrm{CT}$, and can be difficult to detect without intravenous contrast. FDG PET is a useful tool to detect metabolically active disease surrounding the perirenal space (Fig. 10). The infiltrative form presents as bilateral renal enlargement. On FDG PET, it is difficult to interpret this pattern because of the intense activity in the renal collecting system.

There is minimal description to date of the imaging features of leukaemic involvement of the renal parenchyma. ${ }^{25}$ As with lymphoma, primary renal leukaemia is rare. There are two common presentations of renal leukaemia; a diffuse infiltrative type usually presenting with renal failure and nephromegaly, and the other form presenting as focal renal masses (Fig. 11). As renal impairment often develops secondary to the disease process, treatment or glomerulonephritis, FDG PET offers a valuable non-nephorotoxic imaging modality.

\section{Renal metastases}

Renal metastases are rare and often clinically silent. They are small, multicentric and bilateral, ${ }^{26}$ and can be easily missed on conventional CT imaging. Among the
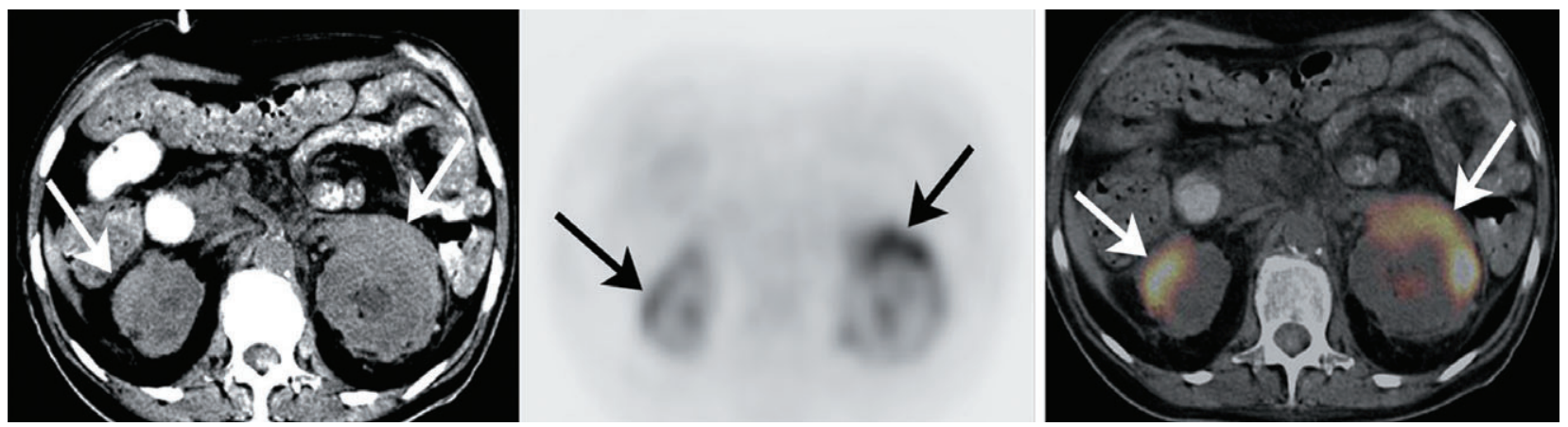

Fig. 10. Fluorine-18 fluoro-2-deoxy-D-glucose (FDG) positron emission tomography (PET)/CT appearances of renal lymphoma. Unenhanced CT image (left) demonstrates bilateral perirenal plaque lesions inseparable from the adjacent renal parenchyma (arrows). PET image (centre) and fused PET/CT image (right) clearly demonstrate increased FDG uptake in these lesions (arrows). Perirenal masses are one of the more typical patterns of renal lymphoma which can be easily identified on metabolic imaging, as an intensely FDG avid lesion. 


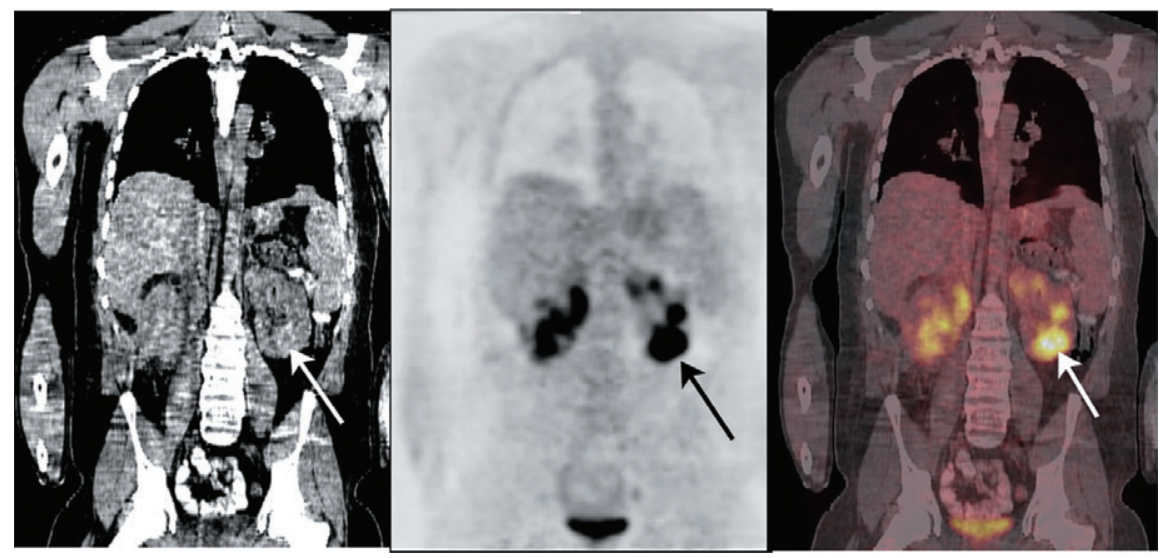

Fig. 11. Fluorine-18 fluoro-2-deoxy-Dglucose (FDG) positron emission tomography (PET)/CT appearances of renal leukaemia. Unenhanced CT image (left) reveals no convincing abnormality. PET image (centre) shows multiple focal areas of intense uptake of FDG, often confused with uptake in the collecting system. Fused image, however, clearly localises these focal areas to the renal cortex with the largest focal lesion seen in the lower pole of the left kidney (arrows), histologically confirmed renal leukaemic deposit. Multifocal renal involvement is one of the two commonest patterns of renal leukaemic involvement.
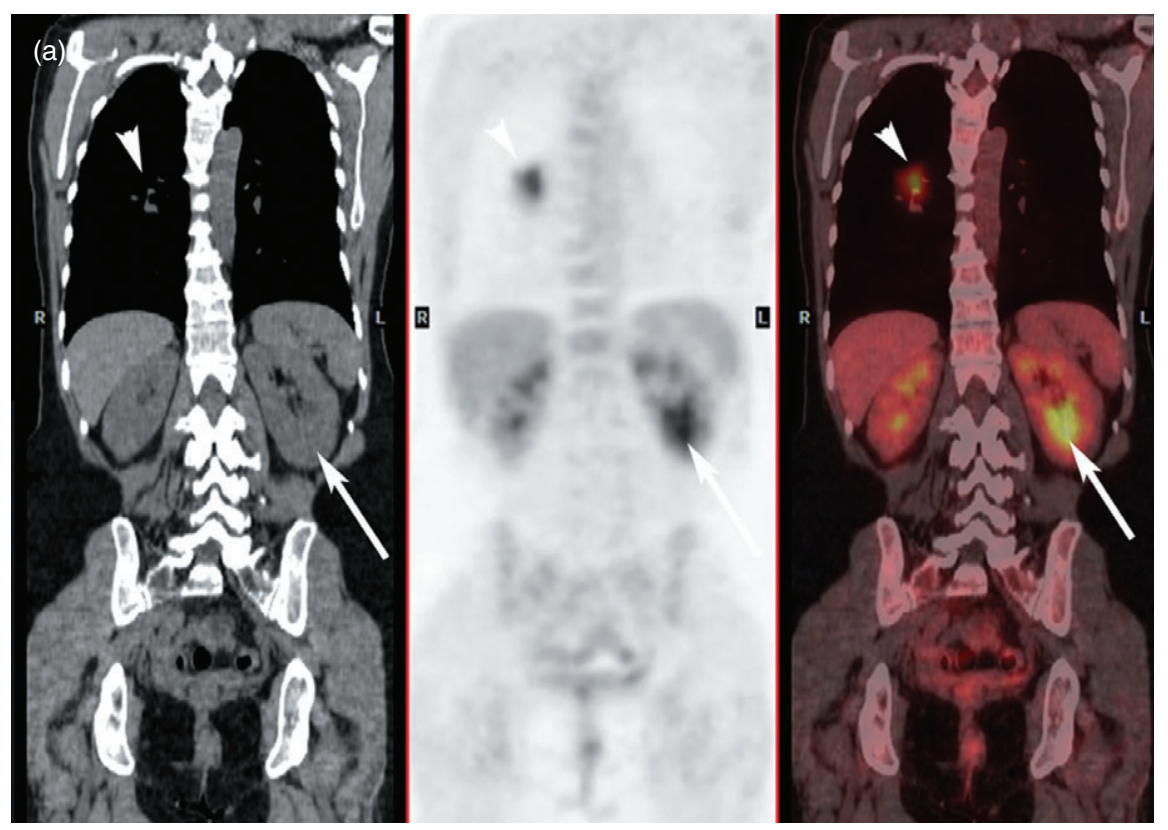

Fig. 12. Fluorine-18 fluoro-2-deoxy-Dglucose (FDG) positron emission tomography (PET)/CT appearances of renal metastases. (a) FDG PET/CT image with coronal CT, attenuation corrected and fused images, performed for suspected solitary pulmonary metastases in a patient with malignant melanoma confirms significant metabolic uptake in a right lung nodule (arrow heads). High-grade uptake is also seen in a focal lesion in the mid pole of the left kidney (arrows) with no convincing abnormality on the unenhanced $\mathrm{CT}$. (b) Axial contrast-enhanced CT done a month later showed rapid increase in size (arrow) and a renal vein thrombus (arrowhead). Post-operative histology confirmed renal metastases from malignant melanoma.

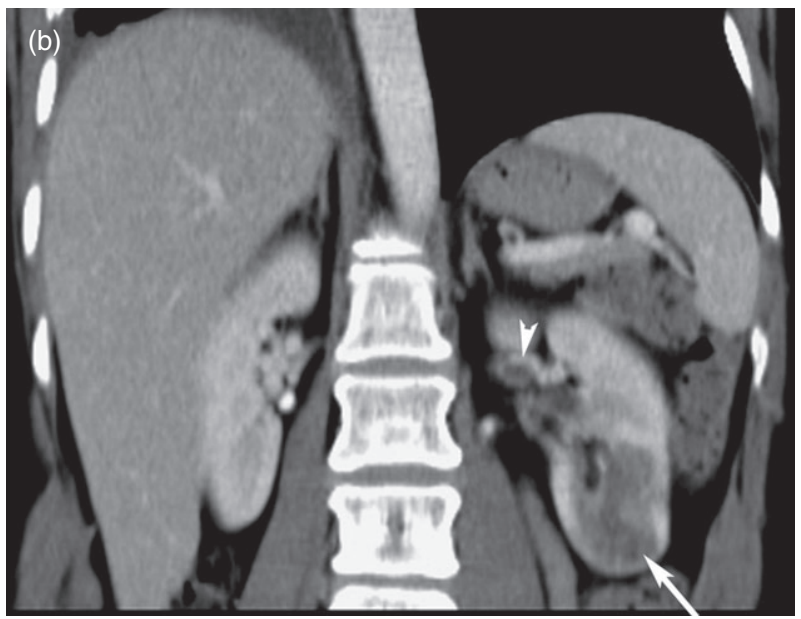


Fig. 13. Fluorine-18 fluoro-2-deoxy-Dglucose (FDG) positron emission tomography (PET)/CT appearances of a potential renal metastasis in a native kidney post-renal transplant. (a) Maximum Intensity Projection (MIP) image (left) demonstrates an FDG avid focus in the right lung (arrow head), histologically confirmed small cell cancer and focus of highgrade uptake in the left renal area (thin arrow). Unenhanced CT image (top right) demonstrates bilateral atrophic native kidneys with a barely perceptible lesion in the left kidney (arrow). A focus of intense FDG uptake is seen in the left kidney on the fused PET/CT (arrow in bottom left image). Note uptake in a transplant kidney in the left iliac fossa (short arrow) on the MIP image. (b) Contrast-enhanced CT confirms an enhancing lesion in the left kidney (arrow), most likely in keeping with renal metastasis in the native left kidney from a primary lung cancer.
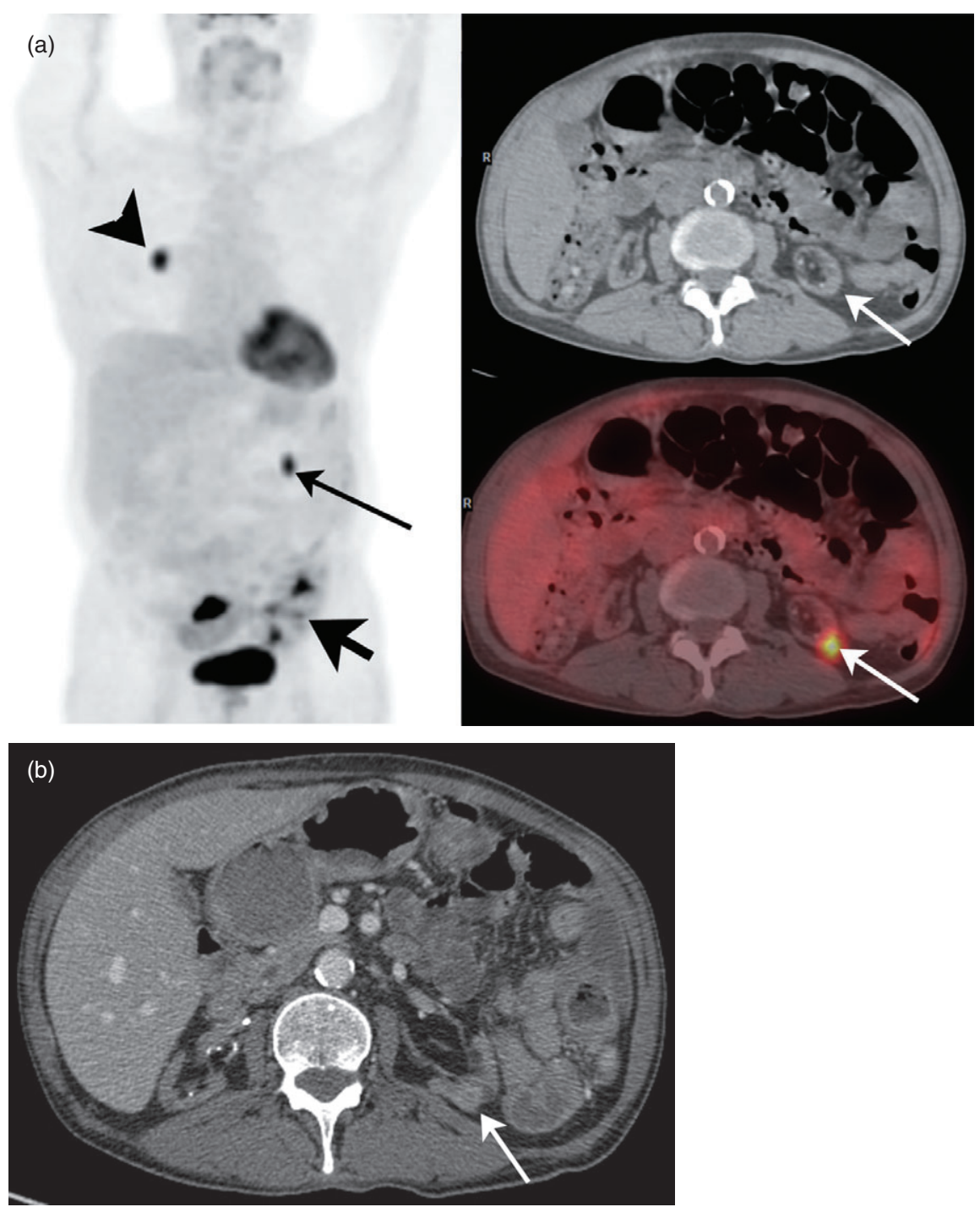

primary tumours that are the most common sources of renal metastases lymphoma, lung cancer and colon cancer show particularly intense FDG activities. Metastases to the kidney from these types of primary malignancies can be detected clearly on FDG PET as hotspots of increased uptake (Figs 12,13). ${ }^{27}$

\section{Discussion}

The advent of hybrid imaging, especially PET/CT, has revolutionised the imaging of oncological diseases. Currently, the role for PET/CT in the work-up of renal lesions is limited. However, with the increasing utilisation of FDG $\mathrm{PET} / \mathrm{CT}$ in cancer, renal lesions are frequently being detected as incidental findings. There is a paucity of literature describing the metabolic appearances of various renal pathologies which has been partly addressed by this review.
Focal-excreted FDG in the renal pelvicalyceal system can often be misinterpreted as potential renal lesions on the PET component, which can be reduced by hydration and use of diuretics. Respiratory misregistration is another factor that needs to be taken into account to avoid misinterpretation. On the other hand, there is a tendency to underestimate disease by mistaking true lesions for normal FDG excretion. Scrutiny of the CT component and fused PET/CT images is needed to minimise both false positive and false negative findings, as illustrated in Figure 6. The importance of viewing the CT component to identify the known morphological features cannot be over-emphasised as the CT component has a significant contribution to lesion characterisation. The CT component of fused PET/CT is often an unenhanced CT; however, PET can now be performed with dedicated contrast-enhanced renal CT as a single PET/CT examination which is an exciting prospect to offer complete 
R Kochhar et al.

Table 2. FDG PET/CT imaging of renal pathologies

\begin{tabular}{|c|c|c|c|}
\hline Renal pathology & Findings on $\mathrm{CT}$ & Findings on FDG PET & Role of FDG PET/CT \\
\hline $\begin{array}{l}\text { Renal angiomyolipoma } \\
\text { (AML) }\end{array}$ & $\begin{array}{l}\text { Detection of macroscopic fat on } \mathrm{CT} \text { is the } \\
\text { key diagnostic feature. }\end{array}$ & $\begin{array}{l}\text { Variable FDG uptake } \\
\text { No role in characterisation }\end{array}$ & $\begin{array}{l}\text { Useful in patients with AML associated } \\
\text { with tuberous sclerosis complex }\end{array}$ \\
\hline Renal oncocytoma & $\begin{array}{l}\text { Iso or Hypodense to renal parenchyma. } \\
\text { Homogenous enhancement with central } \\
\text { stellate scar }\end{array}$ & $\begin{array}{l}\text { Variable FDG uptake } \\
\text { No role in characterisation }\end{array}$ & $\begin{array}{l}\text { Limited role: in particular not useful in } \\
\text { differentiation from renal cell } \\
\text { carcinoma (RCC) }\end{array}$ \\
\hline $\mathrm{RCC}$ & $\begin{array}{l}\text { Hypo, Iso or Hyperdense on unenhanced CT } \\
\text { Significant post-contrast enhancement, at } \\
\text { times heterogeneous as a result of } \\
\text { tumour necrosis }\end{array}$ & $\begin{array}{l}\text { Variable FDG uptake correlated with tumour } \\
\text { grade, GLUT-1 receptors and degree of } \\
\text { tumour necrosis. }\end{array}$ & $\begin{array}{l}\text { Limited role in diagnosis and staging of } \\
\text { primary disease } \\
\text { Useful role in restaging and detection of } \\
\text { metastases }\end{array}$ \\
\hline Renal lymphoma & $\begin{array}{l}\text { Radiological patterns: multiple and solitary } \\
\text { masses, contiguous retroperitoneal } \\
\text { disease, perirenal disease and diffuse } \\
\text { renal infiltration }\end{array}$ & $\begin{array}{l}\text { Foci of increased uptake with distorted renal } \\
\text { parenchymal outline (as opposed to } \\
\text { normal collecting system uptake) }\end{array}$ & $\begin{array}{l}\text { Useful to detect metabolically active } \\
\text { residual and recurrent disease }\end{array}$ \\
\hline Renal leukaemia & $\begin{array}{l}\text { Diffuse infiltrative type seen as } \\
\text { nephromegaly or focal masses }\end{array}$ & $\begin{array}{l}\text { Limited literature. Multifocal type seen as } \\
\text { FDG avid renal lesions }\end{array}$ & $\begin{array}{l}\text { Valuable non-nephorotoxic imaging } \\
\text { modality. }\end{array}$ \\
\hline Renal metastases & $\begin{array}{l}\text { Small, multicentric, bilateral hypodense } \\
\text { lesions. Easily missed on CT }\end{array}$ & Multiple focal FDG avid intrarenal lesions & $\begin{array}{l}\text { Lesions clearly detected with FDG } \\
\text { PET/CT }\end{array}$ \\
\hline
\end{tabular}

FDG, fluorine-18 fluoro-2-deoxy-D-glucose; PET, positron emission tomography.

morphological and metabolic assessment in a single examination.

For accurate characterisation of renal lesions, dedicated ultrasound, contrast-enhanced renal CT and MRI are very useful. Ultrasound and contrast-enhanced CT remain the modalities of choice in renal imaging. The role of MRI in renal imaging is still mainly in differentiating benign lesions versus malignant lesions in patients who cannot undergo CT scanning with intravenous iodinated contrast media, or in cases with non-diagnostic CT results as a problem-solving modality. MRI can have additional diagnostic value in the evaluation of lesions with minimal amounts of fat or with intracellular fat. Data suggest that MRI has a higher sensitivity in evaluating complicated cysts; however, the clinical implications still have to be studied. ${ }^{28}$

The economic impact of the use of PET/CT in characterisation of renal lesions needs consideration. This can be assessed through a cost-effectiveness analysis of different diagnostic strategies performed, for example, in a recent study in which PET/CT was introduced in the clinical management of patients with suspected recurrence of ovarian cancer. ${ }^{29}$ In our experience, PET/CT used in selected high-risk patients, for example in patients with recurrent RCC, can prove to be cost effective by redirecting the clinical management of patients towards more appropriate therapeutic choices.

The imaging features and potential role of PET/CT in some of the common renal lesions have been summarised in Table 2. FDG PET/CT can help in the characterisation of indeterminate cysts, is useful for postoperative surveillance of advanced RCC and can clearly detect FDG avid intraparenchymal renal metastases. $\mathrm{PET} / \mathrm{CT}$ used with other novel metabolites also has a role in the preoperative characterisation of primary RCC and the variability of FDG avidity may also find use in tumour metabolic phenotype determination. The take home messages have been summarised in Table 3 .

\section{Conclusion}

Many different renal lesions can be detected clearly as hotspots of elevated uptake on FDG PET/CT. However, excreted FDG in the urinary tract mimics disease and can interfere with image interpretation. The illustrated cases have demonstrated that by combining careful evaluation of the renal cortex by FDG PET to detect hot spots and correlating it with the anatomical information on the CT component, this modality has the potential to have a role in the imaging of renal lesions. With the increasing use of $\mathrm{PET} / \mathrm{CT}$, a knowledge of both morphological and metabolic characteristics of renal lesions on hybrid imaging is essential to help prevent unnecessary biopsies and ensures optimal management of suspicious lesions.

Table 3. Take home messages

1 Excreted fluorine-18 fluoro-2-deoxy-D-glucose (FDG) in the urinary tract can mimic disease

$2 \mathrm{PET} / \mathrm{CT}$ has a potential role in characterisation of indeterminate renal cysts.

3 Low sensitivity of FDG PET/CT limits its role in detection of primary renal cell carcinoma (RCC) but other novel metabolites can help in the preoperative characterisation of tumour type.

$4 \mathrm{PET} / \mathrm{CT}$ has a useful role in recurrent, metastatic RCC and renal metastases.

$5 \mathrm{PET} / \mathrm{CT}$ has a limited role in the characterisation of renal masses because of variability of uptake, but can be used as a problem-solving modality.

6 Careful scrutiny of the CT component and use of contrast-enhanced CT as a part of the PET/CT is advised. 


\section{References}

1. Terada N, Arai Y, Kinukawa N, Yoshimura K, Terai A. Risk factors for renal cysts. BJU Int 2004; 93 : 1300-02.

2. Goldberg MA, Mayo-Smith WW, Papanicolaou N, Fischman AJ, Lee MJ. FDG PET characterization of renal masses: preliminary experience. Clin Radiol 1997; 52: 510-15.

3. Kaim AH, Burger C, Ganter CC et al. PET-CT-guided percutaneous puncture of an infected cyst in autosomal dominant polycystic kidney disease: case report. Radiology 2001; 221: 818-21.

4. Bissler JJ, Kingswood JC. Renal angiomyolipomata. Kidney Int 2004; 66: 924-34.

5. Israel GM, Bosniak MA. How I do it: evaluating renal masses. Radiology 2005; 236: 441-50.

6. Bachor R, Kotzerke J, Gottfried HW, Brandle E, Reske $\mathrm{SN}$, Hautmann R. [Positron emission tomography in diagnosis of renal cell carcinoma]. Urologe A 1996; 5: $146-50$.

7. Lhommel R, Annet $L$, Bol A et al. PET scan with $11 \mathrm{C}$-acetate for the imaging of liver masses: report of a false positive case. Eur J Nucl Med Mol Imaging 2005; 32: 629.

8. Rintahaka PJ, Chugani HT. Clinical role of positron emission tomography in children with tuberous sclerosis complex. J Child Neurol 1997; 12: 42-52.

9. Choyke PL, Glenn GM, Walther MM, Zbar B, Linehan WM. Hereditary renal cancers. Radiology 2003; 226: 33-46.

10. Noguchi S, Nagashima Y, Shuin T et al. Renal oncocytoma containing 'chromophobe' cells. Int J Urol 1995; 2: 279-80.

11. Blake MA, McKernan M, Setty B, Fischman AJ, Mueller PR. Renal oncocytoma displaying intense activity on 18F-FDG PET. AJR Am J Roentgenol 2006; 186: 269-70.

12. Majhail NS, Urbain JL, Albani JM et al. F-18 fluorodeoxyglucose positron emission tomography in the evaluation of distant metastases from renal cell carcinoma. J Clin Oncol 2003; 21: 3995-4000.

13. Mathew A, Devesa SS, Fraumeni JF Jr, Chow WH. Global increases in kidney cancer incidence, 1973-1992. Eur J Cancer Prev 2002; 11: 171-8.

14. Said JW, Thomas G, Zisman A. Kidney pathology: current classification of renal cell carcinoma. Curr Urol Rep 2002; 3: 25-30.

15. Kang DE, White RL Jr, Zuger JH, Sasser HC, Teigland $\mathrm{CM}$. Clinical use of fluorodeoxyglucose $\mathrm{F} 18$ positron emission tomography for detection of renal cell carcinoma. J Urol 2004; 171: 1806-9.

16. Aide N, Cappele O, Bottet $P$ et al. Efficiency of [(18)F]FDG PET in characterising renal cancer and detecting distant metastases: a comparison with CT. Eur J Nucl Med Mol Imaging 2003; 30: 1236-45.
17. Miyakita $\mathrm{H}$, Tokunaga $\mathrm{M}$, Onda $\mathrm{H}$ et al. Significance of $18 \mathrm{~F}$-fluorodeoxyglucose positron emission tomography (FDG-PET) for detection of renal cell carcinoma and immunohistochemical glucose transporter 1 (GLUT-1) expression in the cancer. Int J Urol 2002; 9: 15-18.

18. Ramdave S, Thomas GW, Berlangieri SU et al. Clinical role of F-18 fluorodeoxyglucose positron emission tomography for detection and management of renal cell carcinoma. J Urol 2001; 166: 825-30.

19. Powles T, Ell PJ. Does PET imaging have a role in renal cancers after all? Lancet Oncol 2007; 8: 279-81.

20. Martinez de Llano SR, Delgado-Bolton RC, Jimenez-Vicioso A et al. [Meta-analysis of the diagnostic performance of 18F-FDG PET in renal cell carcinoma]. Rev Esp Med NuCl 2007; 26: 19-29.

21. Park JW, Jo MK, Lee HM. Significance of 18F-fluorodeoxyglucose positron-emission tomography/computed tomography for the postoperative surveillance of advanced renal cell carcinoma. BJU Int 2009; 103: 615-9.

22. Divgi CR, Pandit-Taskar N, Jungbluth AA et al. Preoperative characterisation of clear-cell renal carcinoma using iodine-124-labelled antibody chimeric G250 (124I-cG250) and PET in patients with renal masses: a phase I trial. Lancet Oncol 2007; 8: 304-10.

23. Da'as N, Polliack A, Cohen $Y$ et al. Kidney involvement and renal manifestations in non-Hodgkin's lymphoma and lymphocytic leukemia: a retrospective study in 700 patients. Eur J Haematol 2001; 67: 158-64.

24. Urban BA, Fishman EK. Renal lymphoma: CT patterns with emphasis on helical CT. Radiographics 2000; 20: $197-212$.

25. Hilmes MA, Dillman JR, Mody RJ, Strouse PJ. Pediatric renal leukemia: spectrum of CT imaging findings. Pediatr Radiol 2008; 38: 424-30.

26. Choyke PL, White EM, Zeman RK, Jaffe MH, Clark LR. Renal metastases: clinicopathologic and radiologic correlation. Radiology 1987; 162: 359-63.

27. Kaneta T, Hakamatsuka T, Yamada T et al. FDG PET in solitary metastastic/secondary tumor of the kidney: a report of three cases and a review of the relevant literature. Ann Nucl Med 2006; 20: 79-82.

28. Nikken JJ, Krestin GP. MRI of the kidney-state of the art. Eur Radiol 2007; 17: 2780-93.

29. Mansueto M, Grimaldi A, Mangili G et al. Positron emission tomography/computed tomography introduction in the clinical management of patients with suspected recurrence of ovarian cancer: a cost-effectiveness analysis. Eur J Cancer Care (Engl) 2009; 18: 612-9. 